\title{
Septic Arthritis of the Pediatric Wrist: A Case Report and Review of the Literature
}

\author{
Jeremy M. Silver $^{1}$, William Hennrikus ${ }^{1}$ \\ 1. Orthopaedic Surgery, Penn State Milton S. Hershey Medical Center, Hershey, USA
}

Corresponding author: Jeremy M. Silver, jeremysilver4@gmail.com

\begin{abstract}
Septic arthritis of the wrist in pediatric patients is a rare diagnosis and is not well described in the literature. We present a case of a 4-month old patient with monoarticular septic arthritis of the wrist treated with surgical drainage and antibiotics. Although a rare diagnosis, septic arthritis of the wrist should be considered in patients with pseudoparalysis of the upper extremity and systemic signs of inflammation. Prompt diagnosis and treatment is critical to prevent permanent damage to the joint. Further data is needed to describe the epidemiology, microbiology, diagnostic findings and treatment of septic arthritis of the pediatric wrist.
\end{abstract}

Categories: Pediatrics, Pediatric Surgery, Orthopedics

Keywords: septic arthritis, wrist, pediatric orthopaedics

\section{Introduction}

Septic arthritis of the wrist in the pediatric population is a rare diagnosis. To our knowledge, there are only three case reports of septic arthritis of the pediatric wrist [1-3]. Acute septic arthritis in a pediatric patient can present in a variety of ways, which can make diagnosis difficult. While there is data regarding the more common pediatric septic arthritis of larger joints, the epidemiology, microbiology, diagnostic criteria and outcomes of septic arthritis of the wrist are not well described. We present a case of a 4-month old with monoarticular septic arthritis of the wrist treated with open surgical drainage and antibiotics.

Received 02/05/2020 Review began 03/04/2020 Review ended 03/26/2020 Published 03/28/2020

๑) Copyright 2020

Silver et al. This is an open access article distributed under the terms of the Creative Commons Attribution License CC-BY 4.0., which permits unrestricted use, distribution, and reproduction in any medium, provided the original author and source are credited.

\section{Case Presentation}

A previously healthy 4-month old male was transferred from an outside hospital for a two-day history of fever and difficulty moving his right arm. There was no history of trauma, insect bites or recent travel. Upon examination, there was mild tenderness and swelling of the right wrist and hand, most notably on the dorsal aspect. The right elbow and shoulder were non-tender with full range of motion. Initial labs showed an erythrocyte sedimentation rate (ESR) of $31 \mathrm{~mm} / \mathrm{hr}$, C-reactive protein (CRP) of $2.45 \mathrm{mg} / \mathrm{dL}$, and white blood cell (WBC) count of $11 \times 103 / \mathrm{mcL}$. The patient was afebrile on admission, although a review of outside records showed one temperature of $101.4^{\circ} \mathrm{F}$ before transfer. $\mathrm{X}$-rays of the right upper extremity showed no evidence of fracture and were not concerning for osteomyelitis.

After initial examination, the case was discussed with the patient's parents and consent was obtained for aspiration of the right wrist with potential irrigation and debridement (I\&D). He was taken to the operating room and placed under general anesthesia. Aspiration of the dorsal aspect of the right wrist joint yielded 1 $\mathrm{mL}$ of pus fluid that was sent to the lab for Gram stain, anaerobic and aerobic cultures. Next, a 2-cm incision was made over the dorsal wrist between the third and fourth extensor compartments (Figure 1). The extensor retinaculum was incised and the extensor tendons were mobilized. Dissection was continued down to the joint capsule and open I\&D of the joint was performed with approximately $200 \mathrm{~mL}$ of sterile saline. The capsule was left open but the skin was closed, and the patient was placed in a soft splint. The patient tolerated the procedure without issue.
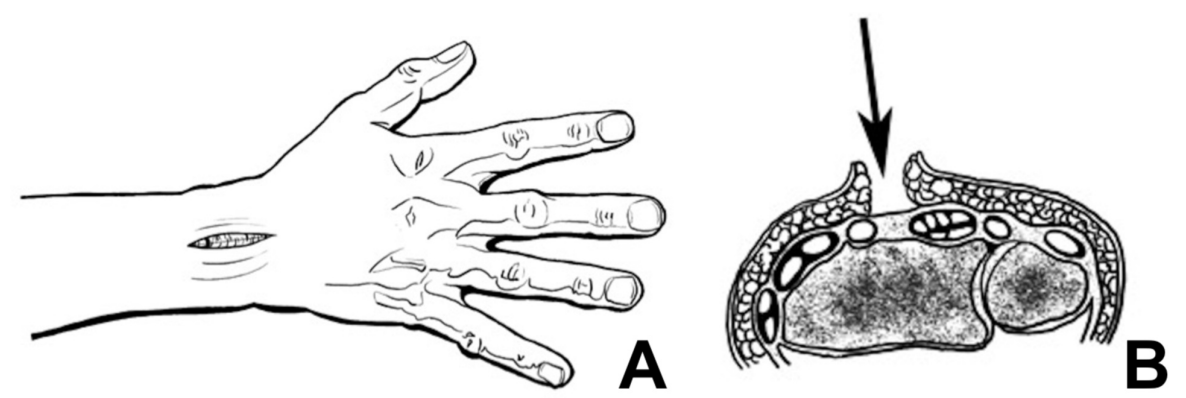


\section{Cureus}

\section{FIGURE 1: Anteroposterior (A) and axial (B) artist renditions depicting the dorsal approach of the wrist between the 3rd and 4th extensor compartments}

Illustrations provided with permission by Julia Hennrikus.

Empiric coverage with IV vancomycin and cefazolin was started. On post-op day two, lab work showed an ESR of $29 \mathrm{~mm} / \mathrm{hr}$, CRP of $1.73 \mathrm{mg} / \mathrm{dL}$ and intra-operative cultures grew methicillin-sensitive Staphylococcus aureus (MSSA) with abundant white cells. Antibiotic treatment was changed to IV nafcillin. On post-op day five, the ESR was $16 \mathrm{~mm} / \mathrm{hr}$, CRP $0.56 \mathrm{mg} / \mathrm{dL}$, and the patient was transitioned to oral cephalexin and rifampin. On post-op day seven, the patient was discharged home on oral antibiotics for a total of four weeks. The patient remained afebrile during the course of the hospital stay and was kept in a soft splint for a total of three weeks. Follow-up appointments at two weeks and three months after discharge showed a wellhealing scar and a normal CRP. Follow-up at one and two years after discharge confirmed no limitations of the right upper extremity with X-ray confirmation of a well-healed wrist joint with open growth plates and no bony abnormalities (Figure 2).

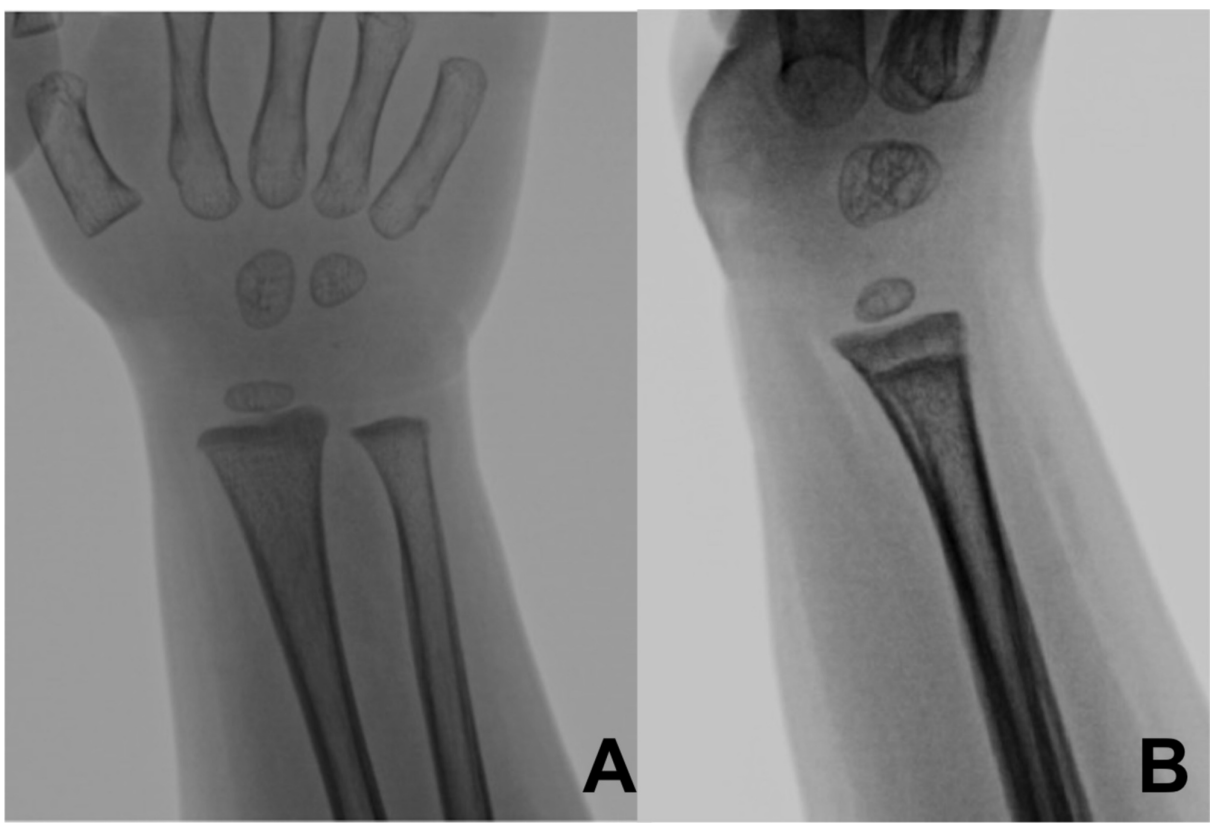

FIGURE 2: Anteroposterior (A) and lateral (B) radiographs of the wrist two years postoperatively with no evidence of bony abnormalities or growth arrest

\section{Discussion}

Septic arthritis of the wrist in the pediatric population is a rare diagnosis. To our knowledge, there have only been three reported cases of pediatric septic arthritis of the wrist in the literature. Wegner et al. in 2017 reported a case of polyarticular arthritis of the left wrist and right knee due to Streptobacillus moniliformis from a rat bite [1]. A retrospective chart review of 40 pediatric patients with septic arthritis by Caksen et al. in 2000 demonstrated only one case of septic arthritis of the wrist [2]. Rahman et al. in 2016 reported a case of septic arthritis of the hand and wrist in an 8-year-old girl due to group A Streptococcus pyogenes treated with surgical drainage [3]. We have presented a case of monoarticular septic arthritis of the wrist in a 4month old patient successfully treated with open surgical drainage and antibiotics.

The differential diagnosis of pediatric patients presenting with pseudoparalysis of the upper extremity is broad. While other infectious etiologies like Lyme disease may be more common, particularly in endemic areas, septic arthritis should be considered as it can result in permanent joint damage with a delay in treatment. Septic arthritis can occur in any joint but more commonly involves the joints of the lower extremity in the pediatric population. Previous studies have shown an overall incidence of acute septic arthritis in the pediatric population between 2 and 13 out of 100,000 in developed countries [4,5]. Of these cases, roughly 80\% involve the knee, hip and ankle with Staphylococcus aureus being the most common 
organism [4-6]. Most cases are caused by hematogenous spread of bacterial pathogens while others can be caused from direct spread via trauma or infection from other neighboring tissues [7]. Prompt diagnosis and treatment is necessary to prevent permanent joint damage and growth plate involvement. Damage is mediated by both host immune response and bacterial toxins in which high cytokine concentrations result in the release of metalloproteinases and other collagen-degrading enzymes [8].

The initial work-up of suspected septic arthritis should include a thorough history, physical exam and labwork, including a complete blood count with differential, ESR, CRP, blood culture, and synovial fluid analysis [5]. Particular emphasis should be placed on obtaining blood cultures as the results will help guide antibiotic treatment. While both ESR and CRP have been shown to be sensitive markers of childhood bone and joint infections, CRP normalizes faster and should be used to monitor recovery [7,9].

Treatment of septic arthritis in pediatric patients begins with prompt diagnosis, surgical drainage, and initiation of antibiotics to prevent permanent damage to the joint [4-7,10]. Empiric antibiotic coverage should be initiated as soon as blood cultures and synovial fluid samples are collected and should include MRSA coverage in endemic areas [4]. Recent studies have shown that short courses of parenteral antibiotics (2-4 days) followed by transition to oral therapy are sufficient to treat uncomplicated cases of septic arthritis [4-6,10]. Specifically, the combination of clinical improvement and a significant reduction in CRP can be used to guide the transition from IV to oral antibiotics [11]. In this case, the patient was treated with five days of IV antibiotics and was transitioned to oral after clinical improvement and a reduction in CRP from $2.45 \mathrm{mg} / \mathrm{dL}$ on admission to $0.56 \mathrm{mg} / \mathrm{dL}$ on post-op day 5 .

The most appropriate surgical treatment of septic arthritis remains open arthrotomy $[4,6]$. Although arthroscopic I\&D has been shown to have success in some cases, the indications for open vs. closed surgical management are not clearly defined in the literature $[4,5]$. Furthermore, the small joint space in pediatric patients limits the feasibility of arthroscopy.

Septic arthritis of the wrist in the adult population is better described in the literature but there are no consensus diagnostic criteria and the true incidence remains unknown $[12,13]$. One recent retrospective chart review by Jennings et al. in 2017 demonstrated a 1.5\% incidence of septic arthritis of the wrist in patients presenting with a swollen, painful wrist without trauma [13]. The most common organism of septic wrist in the adult population is also Staphylococcus aureus [12].

\section{Conclusions}

Septic arthritis of the wrist in pediatric patients is a rare diagnosis but should be considered in any patient presenting with swelling, pain or difficulty moving the wrist and systemic signs of inflammation. We present a case of monoarticular arthritis of the wrist in a pediatric patient successfully treated with appropriate antibiotic use and surgical drainage.

\section{Additional Information}

\section{Disclosures}

Human subjects: Consent was obtained by all participants in this study. Human Subjects Protection Office and Institutional Review Board at Penn State College of Medicine issued approval STUDY00002826. Type of Submission: Initial Study Title of Study: Treatment and Outcomes of Pediatric Septic Arthritis Principal Investigator: Justin Walker Study ID: STUDY00002826 Submission ID: STUDY00002826 Funding: Not Applicable IND,IDE, or HDE: Not Applicable Documents Approved: • HRP-598 - Research Data Plan Review Form 6-18-15.pdf (0.01), Category: IRB Protocol • STUDY 2826 protocol Septic Arthritis 6-18-15.pdf (0.02), Category: IRB Protocol Review Level: Expedited On 6/19/2015, the IRB approved the above-referenced Initial Study. Conflicts of interest: In compliance with the ICMJE uniform disclosure form, all authors declare the following: Payment/services info: All authors have declared that no financial support was received from any organization for the submitted work. Financial relationships: All authors have declared that they have no financial relationships at present or within the previous three years with any organizations that might have an interest in the submitted work. Other relationships: All authors have declared that there are no other relationships or activities that could appear to have influenced the submitted work.

\section{Acknowledgements}

Illustrations by Julia Hennrikus.

\section{References}

1. Wegner AM, Look N, Haus BM: Surgical management of multijoint septic arthritis due to rat-bite fever in a pediatric patient: a case study. Case Rep Orthop. 2017, 1-3. 10.1155/2017/2183941

2. Caksen H, Ozturk MK, Uzum K, Yuksel S, Ustunbas HB, Per H: Septic arthritis in childhood. Pediatr Int. 2000, 42:534-540. 10.1046/j.1442-200x.2000.01267.x

3. Rahman S, Salim F, Khandwala A: Septic arthritis of the hand and wrist in an 8-year-old girl not involving the radiocarpal joint. J Clin Case Rep. 2016, 6:793. 10.4172/2165-7920.1000793 


\section{Cureus}

4. Montgomery NI, Epps HR: Pediatric septic arthritis. Orthop Clin North Am. 2017, 48:209-216. 10.1016/j.ocl.2016.12.008

5. Arnold JC, Bradley JS: Osteoarticular infections in children. Infect Dis Clin North Am. 2015, 29:557-574. 10.1016/j.idc.2015.05.012

6. Dodwell ER: Osteomyelitis and septic arthritis in children: current concepts . Curr Opin Pediatr. 2013, 25:58-63. 10.1097/MOP.0b013e32835c2b42

7. Paakkonen M, Peltola H: Bone and joint infections. Pediatr Clin North Am. 2013, 60:425-436. 10.1016/j.pcl.2012.12.006

8. Shirtliff ME, Mader JT: Acute septic arthritis. Clin Microbiol Rev. 2002, 15:527-544. 10.1128/cmr.15.4.527 544.2002

9. Paakkonen M, Kallio MJ, Kallio PE, Peltola H: Sensitivity of erythrocyte sedimentation rate and c-reactive protein in childhood bone and joint infections. Clin Orthop Relat Res. 2010, 468:861-866. 10.1007/s11999009-0936-1

10. Castellazzi L, Mantero M, Esposito S: Update on the management of pediatric acute osteomyelitis and septic arthritis. Int J Mol Sci. 2016, 17:855. 10.3390/ijms17060855

11. Chou AC, Mahadev A: The use of c-reactive protein as a guide for transitioning to oral antibiotics in pediatric osteoarticular infections. J Pediatr Orthop. 2016, 36:173-177. 10.1097/BPO.0000000000000427

12. Jennings JD, Zielinski E, Tosti R, Ilyas AM: Septic arthritis of the wrist: incidence, risk factors, and predictors of infection. Orthopedics. 2017, 40:e526-e531. 10.3928/01477447-20170404-01

13. Jennings JD, Ilyas AM: Septic arthritis of the wrist. J Am Acad Orthop Surg. 2018, 26:109-115. 10.5435/JAAOS-D-16-00414 